\title{
REFLECTION OF SOLAR ACTIVITY DYNAMICS IN RADIONUCLIDE DATA
}

\author{
A. V. BLINOV and M. N. KREMLIOVSKIJ
}

St. Petersburg Technical University, Polytechnicheskaya 29, St. Petersburg 195251 Russia

\begin{abstract}
Variability of solar magnetic activity manifested within sunspot cycles demonstrates features of chaotic behavior. We have analyzed cosmogenic nuclide proxy records for the presence of the solar activity signals. We have applied numerical methods of nonlinear dynamics to the data showing the contribution of the chaotic component. We have also formulated what kind of cosmogenic nuclide data sets are needed for investigations on solar activity.
\end{abstract}

\section{INTRODUCTION}

Recent advances in nonlinear dynamics make it possible to distinguish stochastic and chaotic behavior of physical systems from long-term observations. Several researchers (Weiss 1988; Morfill et al. 1991) have considered the solar magnetic dynamo as a dissipative system demonstrating temporal variability. The only direct data set long enough for solar activity evolution studies is the sunspot (Wolf index) record covering the period from about AD 1700 until the present. The socalled Packard-Takens procedure transforms this one-dimensional record into the topological equivalent of the phase portrait of the initial dynamic system, thus providing a key for solar magnetic dynamo understanding. Unfortunately, direct solar observations did not continuously cover such an important period of its evolution as the Maunder Minimum. The transition from solar "normal" cycling to the depressed magnetic stage and back would give important information on the nature of and the distinction between chaos and order in solar variability. The only opportunity to prolong the time scale is to use the indirect solar signal reflected in cosmogenic nuclide data. The prospects in the field accounting for the advantages of accelerator mass spectrometry (AMS) in these investigations were discussed earlier (Newkirk 1984). In this paper, we attempt to analyze the representative nuclide series in light of the chaotic nature of solar activity.

\section{CHAOS IN THE SOLAR MAGNETIC CYCLE}

In a sequence of observations, we have used monthly mean data on sunspot numbers (Zürich, 1749-1985, designated below as $\{\mathrm{W}\}$ ). First, we will discuss the assumptions that are made in applying the methods of nonlinear dynamics. We consider the evolution of solar magnetic activity as a temporal chaotic process in a dissipative system. Thus, we assume that its behavior is deterministic, and can be described by combined nonlinear differential equations. The number of equations coincide with the number of system degrees of freedom. A set of scenarios of either chaos or routes to chaos is known (Schuster 1984; Berge, Pomeau \& Vidal 1988). Thus, we can generally predict the shapes of the signal and its spectrum and Poincare map of the phase trajectory for model systems. In this sense, it is possible to compare the observed dynamics with canonical scenarios. Based upon the minimal information, we are able to learn the type of nonlinear equations describing processes occurring in the natural system. We have reconstructed the topology of the phase trajectory of the solar activity from $\{\mathrm{W}\}$ following the Packard-Takens procedure (Packard et al. 1980; Takens 1981), which corresponds to each element of the primary one-dimensional sequence $\{\mathrm{W}\}$ an $n$-dimensional vector $\mathbf{W}_{1}$, in accordance with the rule

$$
\mathrm{W}_{1} \Rightarrow\left(\mathrm{W}_{\mathrm{i}}, \mathrm{W}_{\mathrm{i}+\tau}, \mathrm{W}_{\mathrm{i}+2 \tau}, \ldots, \mathrm{W}_{\mathrm{i}+(\mathrm{n}-1) \tau}\right)
$$

where $\tau$ is a time-delay parameter. The parameter, $\tau$, should be chosen close to the first zero of 
the autocorrelation function for the initial quasi-periodical one-dimensional sequence (we have chosen it equal to 35 months). By the phase space for the dissipative system, we mean the basis formed by several mutually independent physical values.

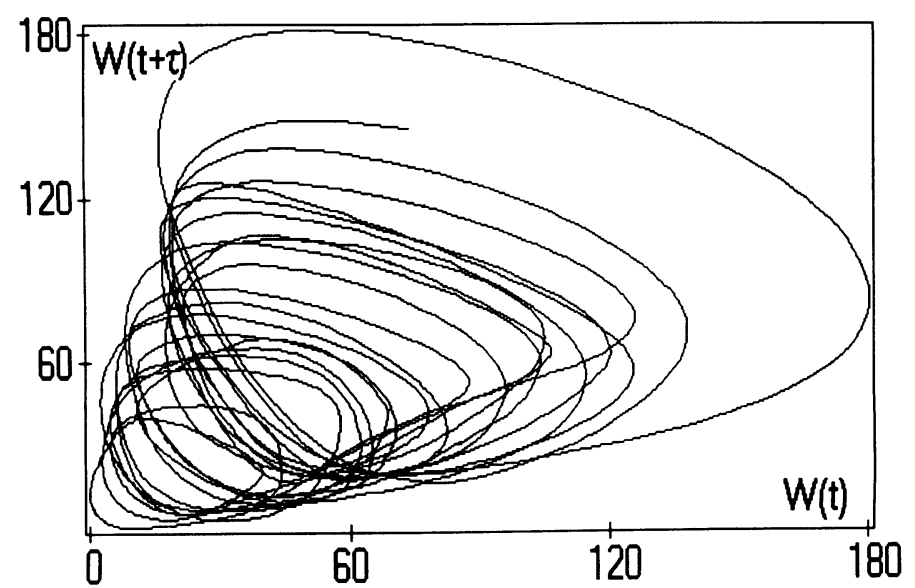

Fig. 1. The two-dimensional projection of the $\{W\}$ trajectory reconstructed in three-dimensional space

Our calculations show that when $\tau$ varies from 30 to 40 months, the basis of the phase space is almost orthogonal. This is the minimal correlation between the neighboring points of the trajectory. The dimension of the embedding space for the phase trajectory may be determined with the help of the Grassberger-Procaccia (1983) algorithm. The analysis of the $\mathbf{W}_{1}$ sequence reveals (Kurtz \& Herzel 1987; Kremliovskij, Blinov \& Tcherviakov 1992) that the dimension of the solar attractor is $<3$. Thus, $n=3$ may be used in the following calculations. Figure 1 shows the trajectory of the solar activity attractor. The high-frequency $(T<\tau)$ noise was subtracted from the primary $\{\mathrm{W}\}$ data before the trajectory reconstruction. The trajectory develops as a broadening coil, one turn corresponding to an 11-yr cycle. The cycles with small maxima lie on the base of the coil. The interval between the trajectory failure at the top to the bottom of the coil corresponds to the $90-\mathrm{yr}$ Gleissberg cycle. The Poincare map (map of the first return) of the trajectory (Fig. 2), which was produced by its crossing by the plane parallel to the coil symmetry axis, contains only 20 points subdividing into 2 groups. The points formed by the first 13 cycles (years before 1900) can be

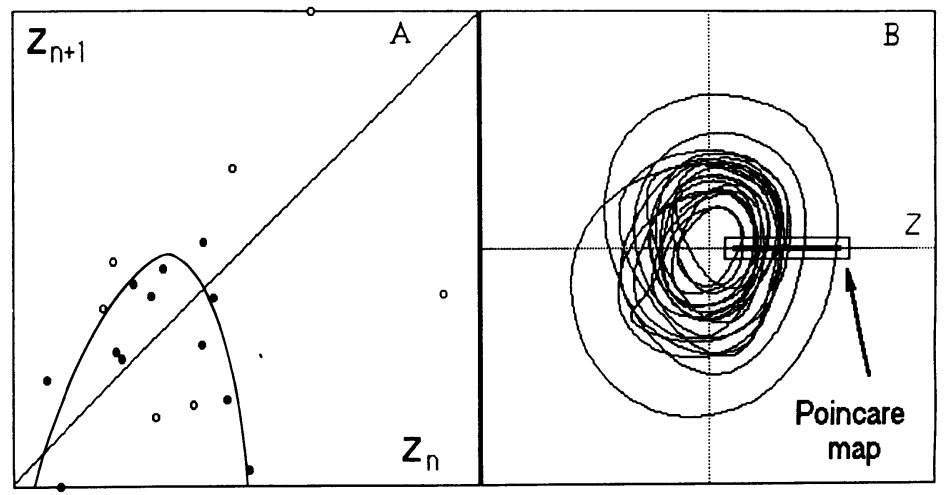

Fig. 2. A. The Poincare map of the phase trajectory: $\bullet=1749-1900 \mathrm{yr} ; \circ=1900-1985 \mathrm{yr}$. B. The $\{\mathrm{W}\}$ phase trajectory turned with its symmetry axis perpendicular to the picture plane; the cross-section plane is shown by the arrow. 
approximated by the quadratic-type curve shown on the graph. It provides evidence for the presence of chaotic dynamics during this stage of solar activity history. The next cycles (14-17) show a different distribution on the map. The causes for this discrepancy do not have a single meaning, but the whole picture supports the presence of chaos in solar activity dynamics. See Kremliovskij, Blinov and Tcherviakov (1992) for further elaboration.

\section{COSMOGENIC NUCLIDE DATA ANALYSIS}

All the reasoning on the chaos in solar activity was obtained from the $\{\mathrm{W}\}$ data. Although the qualitative relationship between the solar magnetic field density reflected in the sunspot index and the production of cosmogenic nuclides in the Earth's atmosphere was experimentally established for ${ }^{14} \mathrm{C}$ and ${ }^{10} \mathrm{Be}$ (Kocharov et al. 1985; Beer et al. 1990), the quantitative model describing their relation has not been developed yet. Transport of the magnetic field from the solar surface to the heliosphere is a complicated process, but one would expect that it is nearly random on the monthly time scale. On the other hand, a simple empirical relationship between $\{\mathrm{W}\}$ and the nuclide mean global production rate can be obtained formally as Blinov (1988) did for the yearly series. Thus, the inevitable problem is the choice of proper time scale, which is closely related to another question, "What are the signal and noise in radionuclide records?" To illustrate this, Figures 3 and 4 show the analysis of the ${ }^{10} \mathrm{Be}$ record in the Dye-3 Greenland ice core (Beer et al. 1990). The

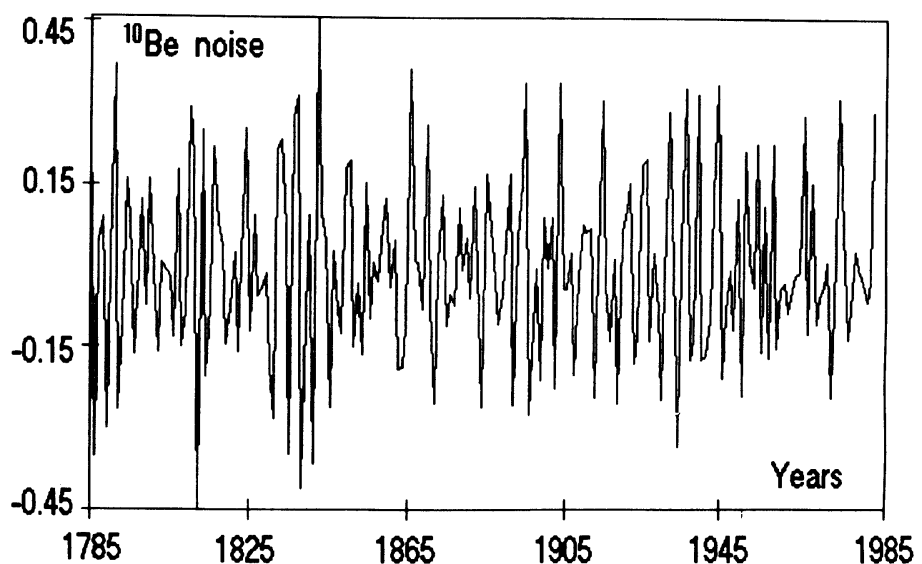

Fig. 3. Noise component of the ${ }^{10} \mathrm{Be}$ Dye-3 (Beer et al. 1990) ice-core record

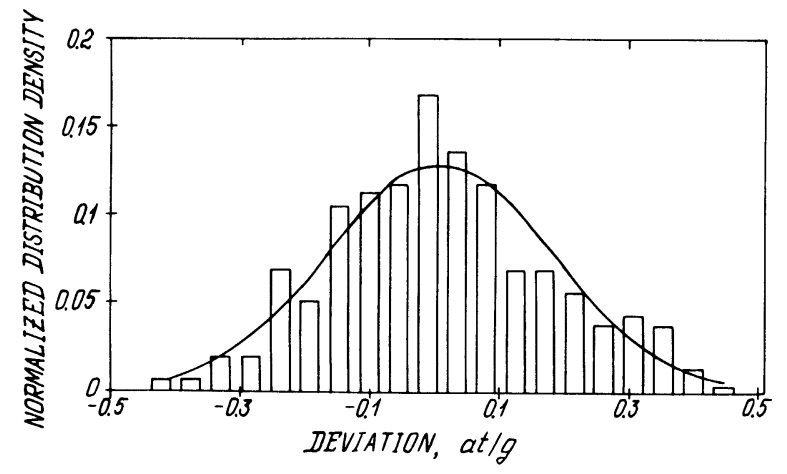

Fig. 4. Histogram of the noise component of ${ }^{10} \mathrm{Be}$ in ice compared with normalized normal distribution 
noise component (Fig. 3) has been extracted from the record by subtracting the smoothed data by a 3-yr moving average. It is clear from Figure 4 that the noise component appears to be consistent with normal distribution, based on the noise mean and dispersion.

The increase of the filter length causes an increase in the discrepancy between the noise and the normal distribution. We interpret this to be a lack of a pronounced physical signal in the record on a time scale of $<3-4 \mathrm{yr}$. We found that the noise component of the ${ }^{14} \mathrm{C} 400$-yr record (Kocharov et al. 1985) fits a normal distribution even better than in Figure 4. This can be explained by natural averaging of ${ }^{14} \mathrm{C}$ yearly concentrations by the natural mixing in the environmental exchange reservoirs. Only additional information would allow us to extract the high-frequency ( $\mathrm{T}<3-4 \mathrm{yr}$ ) component from these data.

Our attempts to reconstruct the phase trajectories of the records mentioned above have not yielded such simple phase portraits as for $\{\mathrm{W}\}$. The dimensions of the embedding phase spaces are much larger than those in Figure 1. We understand this to be the complex influence of nuclide production and transport processes upon the archives, which obviously have their own additional dynamics. Thus, extraction of a pure fine structure of the solar signal from these radionuclide records is not possible with the current level of knowledge of such processes.

The next data set we used is the ${ }^{14} \mathrm{C} 4$ ka tree-ring record (Stuiver \& Becker 1986), consisting of decadal measurements. The map $\delta^{14} \mathrm{C}(\mathrm{t}+\tau)=\mathrm{F}\left[\delta^{14} \mathrm{C}(\mathrm{t})\right]$ with $\tau=1$ (close to one 11 -yr interval) in Figure 5 demonstrates the total lack of memory in the ${ }^{14} \mathrm{C}$ reflection of the Schwabe cycle. Figure 5 should be compared with Figure $2 \mathrm{~A}$, which shows a deterministic relation among the successive $11-y r$ cycles. On the contrary, Figure 5 is more consistent with the map of purely noisy time series, which would show an irregular distribution of the points on the $\{x(t), x(t+\tau)\}$ twodimensional plane. Thus, we conclude that the information on a solar attractor is obscured in this record and cannot complement the sunspot results.

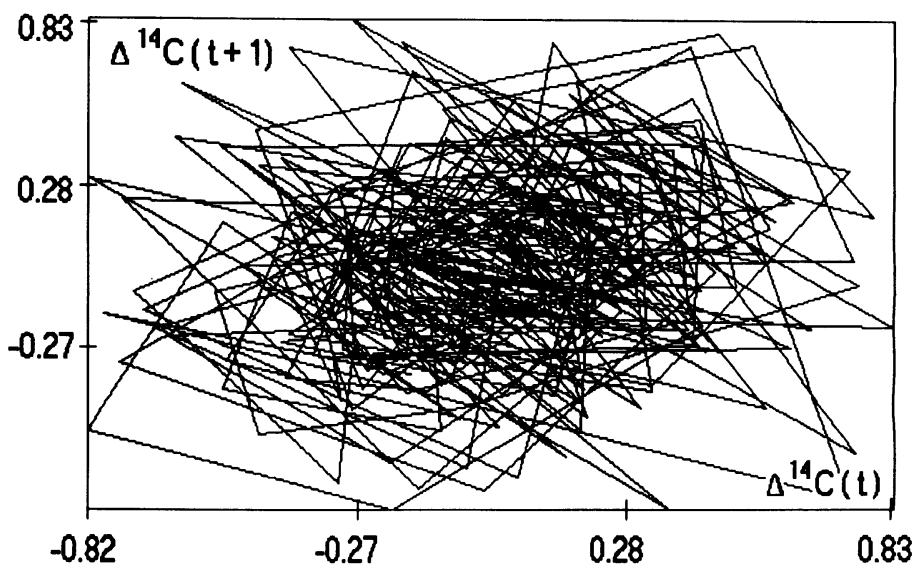

Fig. 5. Two-dimensional map of the ${ }^{14} \mathrm{C}$ record (Stuiver \& Becker 1986) cleared of the minima periods and Gleissberg cycle. The time-delay parameter, $\tau$, is taken to equal $1(10 \mathrm{yr})$.

Finally, we have examined the distribution of solar minima durations in the ${ }^{14} \mathrm{C} 9.7 \mathrm{ka}$ record (Stuiver \& Braziunas 1988). Although the perfect determination of the minima beginnings and endings is arguable, Figure 6 compares this distribution with the predictions of the chaotic system following the type- 1 intermittence route to chaos. The alternation of laminary and chaotic phases 


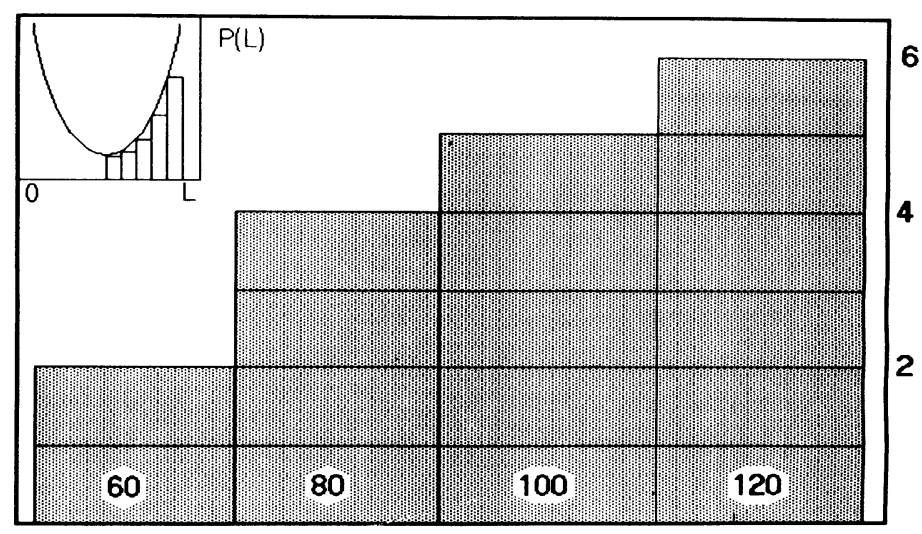

Period of grand minima, years

Fig. 6. The distribution of solar minima durations in the ${ }^{14} \mathrm{C}$ tree-ring record (Stuiver \& Braziunas 1988). Shown in the upper left corner is the distribution of the durations of laminary stages for the type- 1 intermittency route to chaos.

is normally understood under the intermittency in temporal series. Intermittency types are classified according to their Poincare maps, duration of stages, etc. (Berge, Pomeau \& Vidal 1988). Our proposal of type- 1 intermittency agrees with variations in the ${ }^{10} \mathrm{Be}$ concentration in ice during the Maunder Minimum reported by Beer et al. (1991). If solar minima are regular (laminary) periods of magnetic activity evolution, they should preserve the Schwabe-like cyclicity, even though its amplitude may be decreased. Evidence for this view of solar activity will yield new radionuclide data on the periods of quiet Sun.

\section{CONCLUSION}

The results of our work show that cosmogenic nuclides are presently the only source of information on the character of the chaotic behavior of solar activity. The ${ }^{14} \mathrm{C}$ and ${ }^{10} \mathrm{Be}$ proxy records support the view of solar activity evolution as a sequence of chaotic and regular stages, similar to the type-1 intermittency route to chaos. The most promising directions for future studies are the Schwabe-type variations during solar minima and more accurate determination of solar minima durations and transitional behavior.

\section{ACKNOWLEDGMENTS}

We wish to express our gratitude to Dr. N. G. Makarenko for stimulating discussions and interest in our work, and to J. Jirikowic for his careful review of the manuscript and valuable comments. 


\section{REFERENCES}

Beer, J., Blinov, A. V., Bonani, G., Finkel, R. C., Hofmann, H. J., Lehmann, B., Oeschger, H., Sigg, A., Schwander, J., Staffelbach, T., Stauffer, B., Suter, M. and Wölfli, W. 1990 Use of ${ }^{10} \mathrm{Be}$ in polar ice to trace the 11-year cycle of solar activity. Nature 347 : 164-166.

Beer, J., Bonani, G., Dietrich, B., Finkel, R. C., Hofmann, H. J., Lehmann, B. E., Oeschger, H., Stauffer, B., Suter, M. and Wölfli, W. (ms.) 1991 A high resolution ${ }^{10} \mathrm{Be}$ record in polar ice. Paper presented at the 14 th International ${ }^{14} \mathrm{C}$ Conference, Tucson, Arizona, May 20-24, 1991.

Berge, P., Pomeau, Y. and Vidal, C. 1988 L'Order Dans le Chaos. Paris, Editeurs des Science et Arts: 312 p. (in French).

Blinov, A. V. 1988 The dependence of cosmogenic nuclide production rate on solar activity and geomagnetic field variations. In Stephenson, F. R. and Wolfendale, A. W., eds., Secular Solar and Geomagnetic Variations in the Last 10,000 Years. Dordrecht, The Netherlands, Kluwer Academic Publishers: 329-340.

Grassberger, P. and Procaccia, I. 1983 Characterization of strange attractors. Physical Review Letters 50: 346-348.

Kocharov, G. E., Bitvinskas, T. T., Vasilijv, V. A., Dergachev, V. A., Konstantinov, A. N., Metskhvarishvily, R. J., Ostryakov, V. M. and Stupneva, A. V. 1985 Cosmogenic isotopes and astrophysical phenomena. In Astrophysical Phenomena and Radiocarbon. Leningrad: 9-142 (in Russian).

Kremliovskij, M. N., Blinov, A. V. and Tcherviakov, T B. 1992 On the chaotic dynamics of solar activity. Soviet Astronomy, in press (in Russian).

Kurtz, J. and Herzel, H. 1987 An attractor in solar time series. Physica D 25: 165-172.
Morfill, G. E. Scheingraber, H., Voges, W. and Sonett, C. P. 1991 Sunspot number variations: stochastic or chaotic. In Sonett, C., Giampapa, M. and Matthews, M. S., eds., The Sun in Time. Tucson, The University of Arizona Press: 30-58.

Newkirk, G., Jr. 1984 What accelerator mass spectrometry can do for solar physics. In Wölfli, W., Polach, H. A. and Anderson, H. H., eds., Proceedings of the 3rd International Symposium on Accelerator Mass Spectrometry. Nuclear Instruments and Methods B5: 404-410.

Packard, N., Crutchfield, J. P., Farmer, J. D. and Show, R. S. 1980 Geometry from a time series. Physical Review Letters 51: 712-731.

Schuster, H. G. 1984 Deterministic Chaos. Weinheim, Germany, Physics Verlag: $220 \mathrm{p}$.

Stuiver, M. and Becker, B. 1986 High-precision decadal calibration of the radiocarbon time scale, AD 19502500 BC. In Stuiver, M. and Kra, R. S., eds., Proceedings of the 12 th International ${ }^{14} \mathrm{C}$ Conference. Radiocarbon 28(2B): 836-910.

Stuiver, M. and Braziunas, T. F. 1988 The solar component of the atmospheric radiocarbon record. In Stephenson, F. R. and Wolfendale, A. W., eds., Secular Solar and Geomagnetic Variations in the Last 10,000 Years. Dordrecht, The Netherlands, Kluwer Academic Publishers: 245-266.

Takens, F. 1981 Detecting strange attractors in turbulence. In Dynamical Systems and Turbulence. Lectural Notes in Mathematics 898: 336-266.

Weiss, N. O. 1988 Is the solar cycle an example of deterministic chaos? In Stephenson, F. R. and Wolfendale, A. W., eds., Secular Solar and Geomagnetic Variations in the Last 10,000 Years. Dordrecht, The Netherlands, Kluwer Academic Publishers: 69-78. 